\title{
Curvature Corrected Impedance Boundary Conditions in an Arbitrary Basis
}

William T Shaw and Andrew J Dougan 


\section{Curvature Corrected Impedance Boundary Conditions in an Arbitrary Basis}

\begin{abstract}
We present a generalization of the Rytov analysis for curvature corrections to the "impedance boundary condition" (IBC) that allows such boundary conditions to be written down using general coordinate systems and bases for vectors. This removes the need to base the analysis of IBC's on either principal curvature axes or on hypothetical orthogonal coordinate systems. As an application, we show how to write down high-order IBC's for surfaces that are the deformation of a plane. We also show how the use of such an IBC can be used to further reconcile Kirchhoff and small perturbation methods.
\end{abstract}

Index Terms-Approximate boundary condition, impedance, impedance (boundary) condition, Leontovich boundary condition, dielectric body, impedance body, impedance surface, electromagnetic scattering

\section{INTRODUCTION}

A full treatment of the scattering from a dielectric generally involves coupling between the relevant fields through complicated integral equations. Considerable simplification, in the sense that the equations decouple, may be achieved by adopting some form of local boundary condition.

Consider two volumes $V_{1}$ and $V_{2}$ separated by a surface $S$ with unit normal $\bar{n}$ pointing into $V_{1}$. Let radiation be incident on $S$ from sources in $V_{1}$. In the case of a perfect conductor the local boundary condition is exact and given by:

$$
\bar{n} \wedge \bar{E}_{1}=\overline{0}
$$

where $\bar{E}_{1}$ is the electric field in $V_{1}$. To solve the scattering problem one is required to prescribe the surface tangential magnetic field. This may be done in exactly by solving the surface current integral equation (SCIE) for $\bar{n} \wedge \bar{H}_{1}$, or by writing down approximations such as the one given by Kirchhoff [1]:

$$
\bar{n} \wedge \bar{H}_{1}=2 \bar{n} \wedge \bar{H}_{i}
$$

where $\bar{H}_{1}$ is the magnetic field in $V_{1}$ and $\bar{H}_{i}$ is the incident magnetic field. A detailed discussion of the application of this boundary condition to scattering from an ocean-like surface, treated as a perfect conductor, is given in [2].

In the case of finite conductivity matters are not so straightforward. Fortunately, many surfaces of interest are highcontrast media in the sense that the modulus of the complex refractive index $N$ is reasonably large. If we let $V_{1}$ have dielectric and magnetic parameters $\epsilon_{1}$ and $\mu_{1}$, and $V_{2}$ have parameters $\epsilon_{2}$ and $\mu_{2}$ we can write this quantity as

$$
N=\sqrt{\frac{\epsilon_{2} \mu_{2}}{\epsilon_{1} \mu_{1}}} .
$$

This allows one to consider various asymptotic solutions to the boundary condition issue.

The history of such impedance boundary conditions (IBCs) is given, at least in part, by Pelosi and Ufimtsev [3]. Although it is not clear who proposed it first it seems clear that both Shchukin [4] and Leontovich [5] should be given credit for what we shall refer to as the zeroth-order impedance boundary condition:

$$
\bar{n} \wedge \bar{E}_{1}=Z_{2} \bar{n} \wedge\left(\bar{n} \wedge \bar{H}_{1}\right)
$$

on the surface of the scatterer, where $Z_{2}=\sqrt{\mu_{2} / \epsilon_{2}}$ is the impedance of $V_{2}$.

Rytov [6] appears to be the first to improve on this IBC by including higher order effects due to curvature of $S$. Further development of the mathematical theory of the curvaturecorrected IBC is given in [7], [8] and [9]. The work set out by Senior and Volakis in the last of these references is particularly useful in this present context. They based their analysis on an orthogonal curvilinear coordinate system (OCS), and it is the lifting of this restriction that this paper is concerned with. Such a restriction is a significant limitation - indeed, when one can write down an OCS one is well on the way to being able to being able to separate variables in Maxwell's equations and find exact solutions. One needs to be able to write down accurate IBCs in much more general situations.

In Section II we show that the use of a more general basis yields a simple formula for the curvature corrections. In Section III we use this formulation to give explicit expressions for the curvature corrections when $S$ is the deformation of a plane. Note that we work with the Monge representation of the surface and do not invoke an OCS, indeed the the basis used is tailored to scattering rather than a coordinate system. In section IV we show how inclusion of curvature corrections improve the agreement between the half-space theory set out in [10] and perturbation theory. We present our conclusions in Section V.

\section{A. Topological Issues}

Our main area of application is to surfaces that are a deformation of part of a plane, so global topological considerations are generally not a concern in our work, where we base the IBC on a general vector basis for the tangent space. But if we consider topological constraints, and consider a sphere for example, one cannot find any continuous non-zero basis covering the tangent space for all points on the sphere. This is not a flaw in our particular approach but a general difficulty. For example, the coordinate-induced basis using spherical polar coordinates is singular at both the $\mathrm{N}$ and $\mathrm{S}$ pole of the sphere. Neither can one build the basis based on principal curvaturve axes, since these axes are not unique. Indeed, when more that one patch is needed to cover a surface for topological reasons, the ability to work in a general basis makes any calculation on the patch overlaps more straightforward, since our conditions can be written down with respect to both of 
the bases defined on the overlap region. This is therefore an additional strength of our approach.

\section{Generalized Rytov ANALYSis}

In this section we derive a higher order IBC for a medium which takes into account the curvature of the bounding surface. The method of the derivation is almost identical to that given in the book by Senior and Volakis [9], however it differs in one very important respect. The work presented here is applicable to any orthogonal basis for which one of the vectors is taken as parallel to the surface normal. Senior and Volakis tie themselves to a basis derived from an orthogonal coordinate system. Indeed other authors such as Mitzner [11] have restricted themselves even further by relying on an OCS which gives basis vectors coincident with principal curvature axes.

To set things up we review part of the methodology set out in [9]. In $V_{2}$, the electric and magnetic fields are denoted by $\bar{E}_{2}$ and $\bar{H}_{2}$ and Maxwell's equations are:

$$
\bar{\nabla} \wedge \bar{E}_{2}=i \omega \mu_{2} \bar{H}_{2} \quad, \quad \bar{\nabla} \wedge \bar{H}_{2}=-i \omega \epsilon_{2} \bar{E}_{2}
$$

where $\omega$ is the angular frequency of the wave. We will find it convenient, in order to simplify the analysis following, to work with $\sqrt{\epsilon_{2}} \bar{E}_{2}$ and $\sqrt{\mu_{2}} \bar{H}_{2}$. In terms of these, Maxwell's equations can then be written:

$$
\bar{\nabla} \wedge\left(\sqrt{\epsilon_{2}} \bar{E}_{2}\right)+\left(\sqrt{\epsilon_{2}} \bar{E}_{2}\right) \wedge \bar{\nabla}\left(\ln \sqrt{\epsilon_{2}}\right)=i k_{1} N\left(\sqrt{\mu_{2}} \bar{H}_{2}\right)
$$

$\bar{\nabla} \wedge\left(\sqrt{\mu_{2}} \bar{H}_{2}\right)+\left(\sqrt{\mu_{2}} \bar{H}_{2}\right) \wedge \bar{\nabla}\left(\ln \sqrt{\mu_{2}}\right)=-i k_{1} N\left(\sqrt{\epsilon_{2}} \bar{E}_{2}\right)$

with $k_{1}$ the wave number in $V_{1}$. Let us assume that $|N|$ is large in $V_{2}$ and so write it as

$$
N=\frac{w}{q}
$$

where $w$ is a function of position and $q$ is a small parameter. To construct a systematic solution of equations (6) and (7), we write

$$
\begin{aligned}
\sqrt{\epsilon_{2}} \bar{E}_{2} & =\bar{A} e^{i k_{1} \psi / q} \\
\sqrt{\mu_{2}} \bar{H}_{2} & =\bar{B} e^{i k_{1} \psi / q}
\end{aligned}
$$

where $\bar{A}$ and $\bar{B}$ are vector fields to be determined, and $\psi$ is a phase function. Then Maxwell's equations become:

$$
\begin{gathered}
w \bar{B}-\bar{\nabla} \psi \wedge \bar{A}=\frac{q \sqrt{\epsilon_{2}}}{i k_{1}} \bar{\nabla} \wedge\left[\frac{\bar{A}}{\sqrt{\epsilon_{2}}}\right] \\
w \bar{A}+\bar{\nabla} \psi \wedge \bar{B}=-\frac{q \sqrt{\mu_{2}}}{i k_{1}} \bar{\nabla} \wedge\left[\frac{\bar{B}}{\sqrt{\mu_{2}}}\right] .
\end{gathered}
$$

We seek a solution for $\bar{A}$ and $\bar{B}$ in the form:

$$
\begin{aligned}
& \bar{A}=\bar{A}_{0}+q \bar{A}_{1}+q^{2} \bar{A}_{2}+\ldots \\
& \bar{B}=\bar{B}_{0}+q \bar{B}_{1}+q^{2} \bar{B}_{2}+\ldots
\end{aligned}
$$

Senior and Volakis now go on to solve this system using an orthonormal basis induced by coordinates. We now depart from Senior and Volakis's work and attempt to solve the above system using an orthonormal basis $\bar{e}_{\alpha}, \bar{e}_{\beta}, \bar{e}_{\gamma}$ with the property that

$$
\bar{e}_{\gamma}=\bar{n}, \bar{e}_{\alpha}=\bar{e}_{\beta} \wedge \bar{e}_{\gamma}, \bar{e}_{\beta}=\bar{e}_{\gamma} \wedge \bar{e}_{\alpha}, \bar{e}_{\gamma}=\bar{e}_{\alpha} \wedge \bar{e}_{\beta} .
$$

Note that our basis is not necessarily induced by coordinates, so it is more general than the Senior-Volakis system. Note also that, just as with coordinates, this basis will be restricted to a local patch which depends on the geometry of the surface.

\section{A. Zeroth-Order Solution}

Keeping only the zeroth order terms in $q$ gives

$$
\begin{aligned}
& w \bar{B}_{0}-\bar{\nabla} \psi \wedge \bar{A}_{0}=\overline{0} \\
& w \bar{A}_{0}+\bar{\nabla} \psi \wedge \bar{B}_{0}=\overline{0}
\end{aligned}
$$

and elimination of, say, $\bar{B}_{0}$ gives

$$
|\bar{\nabla} \psi|^{2}=w^{2} .
$$

The tangential components of the electric and magnetic fields are continuous over S. Assuming that the external fields are slowly varying (at least compared to $|k N|$ ) outside, they also vary slowly inside $S$. This is only possible if $\psi$ is constant on $S$. So, on $S, \bar{\nabla} \psi$ is normal to $S$. Since it is also of length $w$ it must be $\pm w \bar{n}$. Using our knowledge about the exact solution for scattering from a plane fixes the sign, and we set

$$
\bar{\nabla} \psi=-w \bar{n} .
$$

So that just inside $S$,

$$
\bar{A}_{0}=\bar{n} \wedge \bar{B}_{0}, \quad \bar{B}_{0}=-\bar{n} \wedge \bar{A}_{0} .
$$

In the orthonormal basis adapted to the surface, this becomes

$$
A_{0 \alpha}=-B_{0 \beta}, \quad A_{0 \beta}=B_{0 \alpha}, \quad A_{0 \gamma}=B_{0 \gamma}=0 .
$$

Continuity of the tangential components of the electric and magnetic fields then gives equation (4). This is the standard Shchukin-Leontovich condition, giving the tangential electric field in terms of the leading order magnetic field.

\section{B. First-Order Solution}

Keeping only the first order terms now gives

$$
\begin{gathered}
\bar{B}_{1}+\bar{n} \wedge \bar{A}_{1}=\frac{\sqrt{\epsilon_{2}}}{i w k_{1}} \bar{\nabla} \wedge\left[\frac{\bar{A}_{0}}{\sqrt{\epsilon_{2}}}\right] \\
\bar{A}_{1}-\bar{n} \wedge \bar{B}_{1}=-\frac{\sqrt{\mu_{2}}}{i w k_{1}} \bar{\nabla} \wedge\left[\frac{\bar{B}_{0}}{\sqrt{\mu_{2}}}\right] .
\end{gathered}
$$

Taking components in our basis, the first of these equations gives:

$$
\begin{gathered}
B_{1 \alpha}-A_{1 \beta}=\frac{\sqrt{\epsilon_{2}}}{i w k_{1}} \bar{e}_{\alpha} \cdot \bar{\nabla} \wedge\left[\frac{\bar{A}_{0}}{\sqrt{\epsilon_{2}}}\right] \\
B_{1 \beta}+A_{1 \alpha}=\frac{\sqrt{\epsilon_{2}}}{i w k_{1}} \bar{e}_{\beta} \cdot \bar{\nabla} \wedge\left[\frac{\bar{A}_{0}}{\sqrt{\epsilon_{2}}}\right] \\
B_{1 \gamma}=\frac{\sqrt{\epsilon_{2}}}{i w k_{1}} \bar{e}_{\gamma} \cdot \bar{\nabla} \wedge\left[\frac{\bar{A}_{0}}{\sqrt{\epsilon_{2}}}\right] .
\end{gathered}
$$


The second yields the set:

$$
\begin{gathered}
A_{1 \alpha}+B_{1 \beta}=-\frac{\sqrt{\mu_{2}}}{i w k_{1}} \bar{e}_{\alpha} \cdot \bar{\nabla} \wedge\left[\frac{\bar{B}_{0}}{\sqrt{\mu_{2}}}\right] \\
A_{1 \beta}-B_{1 \alpha}=-\frac{\sqrt{\mu_{2}}}{i w k_{1}} \bar{e}_{\beta} \cdot \bar{\nabla} \wedge\left[\frac{\bar{B}_{0}}{\sqrt{\mu_{2}}}\right] \\
A_{1 \gamma}=-\frac{\sqrt{\mu_{2}}}{i w k_{1}} \bar{e}_{\gamma} \cdot \bar{\nabla} \wedge\left[\frac{\bar{B}_{0}}{\sqrt{\mu_{2}}}\right] .
\end{gathered}
$$

At this point we introduce connection coefficients associated with our basis. We only need to compute the parts associated with the tangential directions, and represent this by the $2 \times 2$ matrix

$$
M_{\mu \lambda}=\bar{e}_{\mu} \cdot \bar{\nabla} \wedge \bar{e}_{\lambda}, \quad \mu, \lambda=\alpha, \beta .
$$

On expanding the right hand side of (27) and using the definition of $M$ we arrive at:

$\sqrt{\mu_{2}} \bar{e}_{\alpha} \cdot \bar{\nabla} \wedge\left[\frac{\bar{B}_{0}}{\sqrt{\mu_{2}}}\right]=M_{\alpha \alpha} B_{0 \alpha}+M_{\alpha \beta} B_{0 \beta}-\sqrt{\mu_{2}} \bar{e}_{\gamma} \cdot \bar{\nabla}\left[\frac{B_{0 \beta}}{\sqrt{\mu_{2}}}\right]$

Similarly equation (28) gives

$\sqrt{\mu_{2}} \bar{e}_{\beta} \cdot \bar{\nabla} \wedge\left[\frac{\bar{B}_{0}}{\sqrt{\mu_{2}}}\right]=M_{\beta \alpha} B_{0 \alpha}+M_{\beta \beta} B_{0 \beta}+\sqrt{\mu_{2}} \bar{e}_{\gamma} \cdot \bar{\nabla}\left[\frac{B_{0 \alpha}}{\sqrt{\mu_{2}}}\right]$

Substituting the expressions (21) for the zeroth order solution into equations (24) and (25) give two slightly different relations. These are:

$\sqrt{\epsilon_{2}} \bar{e}_{\alpha} \cdot \bar{\nabla} \wedge\left[\frac{\bar{A}_{0}}{\sqrt{\epsilon_{2}}}\right]=-M_{\alpha \alpha} B_{0 \beta}+M_{\alpha \beta} B_{0 \alpha}-\sqrt{\epsilon_{2}} \bar{e}_{\gamma} \cdot \bar{\nabla}\left[\frac{B_{0 \alpha}}{\sqrt{\epsilon_{2}}}\right]$

and

$\sqrt{\epsilon_{2}} \bar{e}_{\beta} \cdot \bar{\nabla} \wedge\left[\frac{\bar{A}_{0}}{\sqrt{\epsilon_{2}}}\right]=-M_{\beta \alpha} B_{0 \beta}+M_{\beta \beta} B_{0 \alpha}-\sqrt{\epsilon_{2}} \bar{e}_{\gamma} \cdot \bar{\nabla}\left[\frac{B_{0 \beta}}{\sqrt{\epsilon_{2}}}\right]$.

Note that (25) and (27) represent two independent expressions for $A_{1 \alpha}+B_{1 \beta}$. Equating these gives

$$
\sqrt{\mu_{2}} \bar{e}_{\alpha} \cdot \bar{\nabla} \wedge\left[\frac{\bar{B}_{0}}{\sqrt{\mu_{2}}}\right]=-\sqrt{\epsilon_{2}} \bar{e}_{\beta} \cdot \bar{\nabla} \wedge\left[\frac{\bar{A}_{0}}{\sqrt{\epsilon_{2}}}\right] .
$$

It follows from (31) and (34) that

$$
\begin{aligned}
\sqrt{\mu_{2}} \bar{e}_{\gamma} \cdot \bar{\nabla}\left[\frac{B_{0 \beta}}{\sqrt{\mu_{2}}}\right]=-\frac{1}{2} B_{0 \beta} \bar{e}_{\gamma} \cdot \bar{\nabla}\left(\ln Z_{2}\right) \\
+\frac{1}{2}\left[M_{\alpha \alpha}+M_{\beta \beta}\right] B_{0 \alpha}+\frac{1}{2}\left[M_{\alpha \beta}-M_{\beta \alpha}\right] B_{0 \beta} .
\end{aligned}
$$

By a similar argument, the two independent equations for $B_{1 \alpha}-A_{1 \beta}$ leads to the condition that

$$
\begin{aligned}
& \sqrt{\mu_{2}} \bar{e}_{\gamma} \cdot \bar{\nabla}\left[\frac{B_{0 \alpha}}{\sqrt{\mu_{2}}}\right]=-\frac{1}{2} B_{0 \alpha} \bar{e}_{\gamma} \cdot \bar{\nabla}\left(\ln Z_{2}\right) \\
& +\frac{1}{2}\left[M_{\alpha \beta}-M_{\beta \alpha}\right] B_{0 \alpha}-\frac{1}{2}\left[M_{\beta \beta}+M_{\alpha \alpha}\right] B_{0 \beta} .
\end{aligned}
$$

These relations allow us to eliminate the off-surface derivatives involved in computing the components of the curl of $\bar{B}_{0} / \sqrt{\mu_{2}}$. We can combine our results neatly by writing

$$
\sqrt{\mu_{2}} \bar{e}_{\alpha} \cdot \bar{\nabla} \wedge\left[\frac{\bar{B}_{0}}{\sqrt{\mu_{2}}}\right]=R_{\alpha \alpha} B_{0 \alpha}+R_{\alpha \beta} B_{0 \beta}
$$

$$
\sqrt{\mu_{2}} \bar{e}_{\beta} \cdot \bar{\nabla} \wedge\left[\frac{\bar{B}_{0}}{\sqrt{\mu_{2}}}\right]=R_{\beta \alpha} B_{0 \alpha}+R_{\beta \beta} B_{0 \beta}
$$

where

$$
\begin{gathered}
R_{\alpha \alpha}=\frac{1}{2}\left[M_{\alpha \alpha}-M_{\beta \beta}\right] \\
R_{\beta \beta}=\frac{1}{2}\left[M_{\beta \beta}-M_{\alpha \alpha}\right] \\
R_{\alpha \beta}=\frac{1}{2}\left[M_{\alpha \beta}+M_{\beta \alpha}+\bar{e}_{\gamma} \cdot \bar{\nabla}\left(\ln Z_{2}\right)\right] \\
R_{\beta \alpha}=\frac{1}{2}\left[M_{\alpha \beta}+M_{\beta \alpha}-\bar{e}_{\gamma} \cdot \bar{\nabla}\left(\ln Z_{2}\right)\right] .
\end{gathered}
$$

Substituting (38) and (39) into the expressions (27) and (28) for the first-order components leads to:

$$
\begin{gathered}
A_{1 \alpha}=-B_{1 \beta}-\frac{1}{i w k_{1}}\left\{R_{\alpha \alpha} B_{0 \alpha}+R_{\alpha \beta} B_{0 \beta}\right\} \\
A_{1 \beta}=B_{1 \alpha}-\frac{1}{i w k_{1}}\left\{R_{\beta \alpha} B_{0 \alpha}+R_{\beta \beta} B_{0 \beta} \cdot\right\}
\end{gathered}
$$

The zeroth and first order relations we have derived can now be converted into a relationship between the components of the total electric and magnetic fields on the surface. Continuity of the tangential parts of the electric and magnetic fields across the surface gives:

$$
\begin{aligned}
& E_{\alpha}=-Z_{2}\left[H_{\beta}-\frac{1}{i k_{1} N}\left\{R_{\alpha \alpha} H_{\alpha}+R_{\alpha \beta} H_{\beta}\right\}\right]+O\left(N^{-2}\right) \\
& E_{\beta}=Z_{2}\left[H_{\alpha}-\frac{1}{i k_{1} N}\left\{R_{\beta \alpha} H_{\alpha}+R_{\beta \beta} H_{\beta}\right\}\right]+O\left(N^{-2}\right) .
\end{aligned}
$$

This curvature corrected impedance boundary condition can be recast in vectorial form as

$$
\bar{n} \wedge \bar{E}_{1}=Z_{2}\left[\overline{\bar{I}}+\frac{1}{i k_{1} N} \overline{\bar{R}}\right] \cdot \bar{n} \wedge\left(\bar{n} \wedge \bar{H}_{1}\right)
$$

where $\overline{\bar{I}}$ is the unit dyad and

$$
\overline{\bar{R}}=R_{\alpha \alpha} \bar{e}_{\alpha} \bar{e}_{\alpha}+R_{\alpha \beta} \bar{e}_{\alpha} \bar{e}_{\beta}+R_{\beta \alpha} \bar{e}_{\beta} \bar{e}_{\alpha}+R_{\beta \beta} \bar{e}_{\beta} \bar{e}_{\beta} .
$$

Note that (48) gives an impedance boundary condition that is accurate to $O\left(N^{-1}\right)$. This is by no means the first such expression but we believe its usefulness lies in the fact that it involves only the tangental components of the fields and that it is expressed in a general orthonormal basis. Other first-order expressions exist in the literature. For example Artola and Cessenat [12] use a coordinate system parallely transported along $\bar{n}$ to derive a condition that involves the normal derivative of the tangental fields. Senior and Volakis [9] use a basis associated with an OCS to derive a first order solution similar to (48) and have gone further by deriving an expression for terms that are higher order than $N^{-1}$. It is clear that analogous higher order boundary conditions could be obained by pursuing this present analysis further. This would involve higher order derivatives of the electric and magnetic fields and of the orthonormal basis. We leave this for future work. Results for the special case of a periodic boundary to a highly conducting body have been given in [13]. Our results do not require periodicity constraints. 
In the remaining sections we demonstate the ready applicabilty of (48) by using it to calculate the scattering from a surface that is the deformation of a plane. In the next section we begin this process by fixing a non-coordinate basis so as to calculate the $R$-matrix.

\section{Surfaces that are the Deformation of a Plane}

In this section we apply the above theory to the case where $S$ is a deformation of the $z=0$ plane so that $V_{1}$ and $V_{2}$ represent "upper" and "lower" half-spaces. We shall also restrict ourselves to homogeneous materials, that is materials for which the dielectric an magnetic parameters are constants.

We adopt the $(x, y, \eta)$ (Monge) representation of the bounding surface where we will write the Cartesian basis vectors as $\left(\bar{e}_{x}, \bar{e}_{y}, \bar{e}_{z}\right)$ and where $\eta=\eta(x, y)$ is the height above the $z=0$ plane. To facilitate the calculation of the $R$-matrix we choose a basis adapted to $S$ and the incident radar vector $\bar{\kappa}$ in the upper half-space. This is:

$$
\bar{e}_{\alpha}=\frac{(\bar{\kappa} \wedge \bar{n}) \wedge \bar{n}}{|\bar{\kappa} \wedge \bar{n}|}, \bar{e}_{\beta}=\frac{(\bar{\kappa} \wedge \bar{n})}{|\bar{\kappa} \wedge \bar{n}|}, \bar{e}_{\gamma}=\bar{n} .
$$

Inspection of the details of these vectors shows that this basis is slightly problematic around normal incidence. However, the curvature analysis that follows will turn out to be insensitive to this issue.

The surface normal is given by

$$
\bar{n}=T^{-1}\left[-\eta_{x} \bar{e}_{x}-\eta_{y} \bar{e}_{y}+\bar{e}_{z}\right]
$$

where $T=\sqrt{1+\eta_{x}^{2}+\eta_{y}^{2}}$ and where $\eta_{x}$ and $\eta_{y}$ are the first derivatives of $\eta$ with respect to $x$ and $y$ respectively. We shall also take $\bar{\kappa}$ in the $y=0$ plane and incident from the right so that

$$
\bar{\kappa}=-k\left[\sin \theta \bar{e}_{x}+\cos \theta \bar{e}_{z}\right]
$$

where $\theta$ is the incident angle measured from normal incidence to the $z=0$ plane. Hence the orthonormal basis given by (50) is:

$$
\begin{gathered}
\bar{e}_{\alpha}=\frac{1}{T Q}\left[\begin{array}{c}
\sin \theta+\eta_{x} \cos \theta+\eta_{y}^{2} \sin \theta \\
-\eta_{x} \eta_{y} \sin \theta+\eta_{y} \cos \theta \\
\eta_{y}^{2} \cos \theta+\eta_{x}\left(\sin \theta+\eta_{x} \cos \theta\right)
\end{array}\right] \\
\bar{e}_{\beta}=\frac{1}{Q}\left[\begin{array}{c}
-\eta_{y} \cos \theta \\
\sin \theta+\eta_{x} \cos \theta \\
\eta_{y} \sin \theta
\end{array}\right]
\end{gathered}
$$

where $Q=\sqrt{n_{y}^{2}+\left(\sin \theta+\eta_{x} \cos \theta\right)^{2}}$. We shall now calculate $M_{\mu \lambda}$ in this basis for a 2-dimensional corrugated surface and for a 2-dimensional surface for which the surface slopes are small. The first example allows us to check that our analysis is consistent with that for the one-dimensional case discussed by Ngo and Rino [14]. The second allows us to calculate the scattering properties of a rough surface in the LSV approximation [10].

\section{A. Consistency with One-Dimensional Analysis}

We can make contact with the 1-D analysis of Ngo and Rino if we consider a surface that varies only in the $\mathrm{x}$-direction. Then $n_{y}=0$ and

$$
\begin{gathered}
\bar{e}_{\alpha}=T^{-1}\left[\bar{e}_{x}+\eta_{x} \bar{e}_{z}\right] \\
\bar{e}_{\beta}=\bar{e}_{y} \\
\bar{e}_{\gamma}=T^{-1}\left[-\eta_{x} \bar{e}_{x}+\bar{e}_{z}\right] .
\end{gathered}
$$

Since $\bar{\nabla} \wedge \bar{e}_{\beta}=\overline{0}$ this immediately gives us $M_{\alpha \beta}=M_{\beta \beta}=0$. To treat $M_{\alpha \alpha}$, note that

$$
\begin{aligned}
M_{\alpha \alpha} & =\bar{e}_{\alpha} \cdot \bar{\nabla} \wedge \bar{e}_{\alpha}=\bar{e}_{\alpha} \cdot \bar{\nabla} \wedge\left(\bar{e}_{\beta} \wedge \bar{e}_{\gamma}\right)=\bar{e}_{\alpha} \cdot \bar{\nabla} \wedge\left(\bar{e}_{y} \wedge \bar{n}\right) \\
& =\bar{e}_{\alpha} \cdot\left[\bar{e}_{y}(\bar{\nabla} \cdot \bar{n})-\bar{n}\left(\bar{\nabla} \cdot \bar{e}_{y}\right)+(\bar{n} \cdot \bar{\nabla}) \bar{e}_{y}-\left(\bar{e}_{y} \cdot \bar{\nabla}\right) \bar{n}\right]=0
\end{aligned}
$$

To find $M_{\beta \alpha}$ note that:

$$
\begin{gathered}
M_{\beta \alpha}=\bar{e}_{\beta} \cdot \bar{\nabla} \wedge \bar{e}_{\alpha}=\bar{\nabla} \cdot\left(\bar{e}_{\alpha} \wedge \bar{e}_{\beta}\right)+\bar{e}_{\alpha} \cdot \bar{\nabla} \wedge \bar{e}_{\beta} \\
=\bar{\nabla} \cdot \bar{n}+M_{\alpha \beta}=\bar{\nabla} \cdot \bar{n}
\end{gathered}
$$

and hence

$$
M_{\beta \alpha}=\frac{\partial}{\partial x}\left[\frac{1}{\left(1+n_{x}^{2}\right)^{1 / 2}}\right]=-\frac{\eta_{x x}}{T^{3}} .
$$

Calculation of the corresponding $R$-matrix and substitution into our boundary condition leads to the following:

$$
\begin{aligned}
\frac{E_{\alpha}}{H_{\beta}} & =-Z_{2}\left[1-\frac{i \eta_{x x}}{2 k N T^{3}}\right] \\
\frac{E_{\beta}}{H_{\alpha}} & =Z_{2}\left[1+\frac{i \eta_{x x}}{2 k N T^{3}}\right] .
\end{aligned}
$$

As promised these relations are consistent with those of Ngo and Rino [14], to order $N^{-1}$.

\section{B. Two-Dimensional Surfaces in the LSV Approximation}

The Linear Slope Variation (LSV) approximation [10] was developed to calculate the scattering properties of a twodimensional surface when its slopes are small. In this section we calculate the $R$-matrix under this assumption.

According to the LSV approximation we ignore all terms quadratic in the derivatives of $\eta$ and hence, to this order:

$$
\begin{gathered}
\bar{e}_{\alpha} \sim \bar{e}_{x}+\eta_{y} \cot \theta \bar{e}_{y}+\eta_{x} \bar{e}_{z} \\
\bar{e}_{\beta} \sim-\eta_{y} \cot \theta \bar{e}_{x}+\bar{e}_{y}+\eta_{y} \bar{e}_{z} \\
\bar{e}_{\gamma} \sim-\eta_{x} \bar{e}_{x}-\eta_{y} \bar{e}_{y}+\bar{e}_{z} .
\end{gathered}
$$

Therefore we have:

$$
\begin{aligned}
& \bar{\nabla} \wedge \bar{e}_{\alpha} \sim \eta_{x y} \bar{e}_{x}-\eta_{x x} \bar{e}_{y}+\eta_{y x} \cot \theta \bar{e}_{z} \\
& \bar{\nabla} \wedge \bar{e}_{\beta} \sim \eta_{y y} \bar{e}_{x}-\eta_{y x} \bar{e}_{y}+\eta_{y y} \cot \theta \bar{e}_{z} .
\end{aligned}
$$

Hence we obtain:

$$
\begin{gathered}
M_{\alpha \alpha}=\bar{e}_{\alpha} \cdot \bar{\nabla} \wedge \bar{e}_{\alpha} \sim \eta_{x y} \\
M_{\beta \beta}=\bar{e}_{\beta} \cdot \bar{\nabla} \wedge \bar{e}_{\beta} \sim-\eta_{y x} \\
M_{\alpha \beta}=\bar{e}_{\alpha} \cdot \bar{\nabla} \wedge \bar{e}_{\beta} \sim \eta_{y y}
\end{gathered}
$$




$$
M_{\beta \alpha}=\bar{e}_{\beta} \cdot \bar{\nabla} \wedge \bar{e}_{\alpha} \sim-\eta_{x x} .
$$

The $R$-matrix can then be written

$$
R \sim\left[\begin{array}{cc}
\eta_{x y} & \frac{1}{2}\left(\eta_{y y}-\eta_{x x}\right) \\
\frac{1}{2}\left(\eta_{y y}-\eta_{x x}\right) & -\eta_{x y}
\end{array}\right]
$$

where the first column/row refers to the $\alpha$ index and the second column/row refers to the $\beta$ index. This completes our approximate analysis of the $R$-matrix, and we now move on to consider the implications for radar cross section analysis.

\section{Curvature Corrections For the Root RCS}

In [10] we used a half-space Green's function along with the tangent plane approximation for the surface fields to calculate the root RCS of a rough dielectric surface. We were able to show that the resultant expression was consistent with Valenzuela's dielectric perturbation theory [15] to $O\left(N^{-1}\right)$ in the refractive index. In this section we prove the assertion made there that the agreement can be improved to $O\left(N^{-2}\right)$.

\section{A. Recap of Half-Space Theory}

To write down the expressions for the root RCS and the surface fields we introduce the standard polar coordinates $(r, \theta, \phi)$ and their associated orthogonal unit vectors $\left(\bar{u}_{r}, \bar{u}_{\theta}, \bar{u}_{\phi}\right)$. Let $\bar{k}=\bar{k}_{H}+k_{z} \bar{e}_{z}$ be the outgoing wave vector. We must also define the Fresnel reflection coefficients:

$$
\begin{array}{r}
R^{T E}(\psi)=[\cos \psi-s(\psi)] /[\cos \psi+s(\psi)] \\
R^{T M}(\psi)=-[\epsilon \cos \psi-s(\psi)] /[\epsilon \cos \psi+s(\psi)]
\end{array}
$$

and their linear deviations:

$$
\begin{array}{r}
R_{1}^{T E}=2[1-\epsilon] \sin \theta /\left(s(\theta)[\cos \theta+s(\theta)]^{2}\right) \\
R_{1}^{T M}=-2 \epsilon[1-\epsilon] \sin \theta /\left(s(\theta)[\epsilon \cos \theta+s(\theta)]^{2}\right)
\end{array}
$$

where we have taken with $\mu_{1}=\mu_{2}=\mu_{0}, \epsilon=\epsilon_{2} / \epsilon_{1}$ is the relative permittivity and $s(\psi)=\left[\epsilon-\sin ^{2} \psi\right]^{1 / 2}$. Here $\psi$ is the local angle of incidence between the incident wave vector $\bar{\kappa}$ and the unit normal $\bar{n}$ to the surface so that $\cos \psi=$ $-(\bar{\kappa} \cdot \bar{n}) /|\bar{\kappa}|$. Note that we will just write $R^{T E}$ and $R^{T M}$ when considering the flat $z=0$ surface with $\bar{n}=\bar{e}_{z}$ and $\bar{\kappa}=-\bar{k}$.

The half-space expression for the normalized root RCS is

$$
\begin{gathered}
\bar{\Sigma}=\frac{i k}{B_{0}} \int_{S} d S e^{-i \bar{k} \cdot \bar{r}}\left\{\left(\bar{u}_{\phi} \bar{\gamma}-\bar{u}_{\theta} \bar{\alpha}\right) \cdot\left[\bar{n} \wedge \bar{E}_{1}(\bar{r})\right] / c\right. \\
\left.+\left(\bar{u}_{\phi} \bar{\delta}-\bar{u}_{\theta} \bar{\beta}\right) \cdot\left[\bar{n} \wedge \bar{B}_{1}(\bar{r})\right]\right\}
\end{gathered}
$$

where $\bar{B}_{1}=\mu_{1} \bar{H}_{1}, B_{0}$ is the modulus of the incoming magnetic field, $c$ is the speed of light in $V_{1}$ and where $\bar{\alpha}=\left[\bar{u}_{\theta}-e^{2 i k_{z} \eta} R^{T E} \hat{\bar{u}}_{\theta}\right], \bar{\beta}=\left[1+e^{2 i k_{z} \eta} R^{T E}\right] \bar{u}_{\phi}, \bar{\gamma}=$ $-\left[1-e^{2 i k_{z} \eta} R^{T M}\right] \bar{u}_{\phi}, \bar{\delta}=\left[\bar{u}_{\theta}+e^{2 i k_{z} \eta} R^{T M} \hat{\bar{u}}_{\theta}\right]$ and $\hat{\bar{u}}_{\theta}=$ $\bar{u}_{\theta}-2\left(\bar{u}_{\theta} \cdot \bar{e}_{z}\right) \bar{e}_{z}$.

The quantities $\bar{n} \wedge \bar{E}_{1}$ and $\bar{n} \wedge \bar{B}_{1}$ are the unknown surface currents. In the tangent plane approximation they are given by:

$$
\begin{gathered}
\bar{n} \wedge \bar{E}_{1}=-\frac{\left[R^{T E}(\psi)-R^{T M}(\psi)\right]}{|\bar{n} \wedge \bar{\kappa}|^{2}} \bar{n} \cdot\left(\bar{\kappa} \wedge \bar{E}_{i}\right)[\bar{\kappa}-(\bar{n} . \bar{\kappa}) \bar{n}] \\
+\left[1+R^{T M}(\psi)\right] \bar{n} \wedge \bar{E}_{i}
\end{gathered}
$$

$$
\begin{gathered}
\bar{n} \wedge \bar{B}_{1}=-\frac{\left[R^{T E}(\psi)-R^{T M}(\psi)\right]}{|\bar{n} \wedge \bar{\kappa}|^{2}} \bar{n} \cdot\left(\bar{\kappa} \wedge \bar{B}_{i}\right)[\bar{\kappa}-(\bar{n} . \bar{\kappa}) \bar{n}] \\
+\left[1-R^{T E}(\psi)\right] \bar{n} \wedge \bar{B}_{i}
\end{gathered}
$$

where $\bar{E}_{i}$ and $\bar{B}_{i}$ are the incident electric and magnetic fields respectively.

We will restrict ourselves to the case of backscatter, so that $\bar{k}=-\bar{\kappa}$. Let $E_{0}=c B_{0}$. For transmitted horizontal polarization we have an incoming wave of the form

$$
\bar{E}_{i}=-E_{0} \bar{u}_{\phi} e^{-i \bar{k} \cdot \bar{r}}, \quad \bar{B}_{i}=-B_{0} \bar{u}_{\theta} e^{-i \bar{k} \cdot \bar{r}}
$$

For transmitted vertical polarization we have an incoming wave of the form

$$
\bar{E}_{i}=-E_{0} \bar{u}_{\theta} e^{-i \bar{k} \cdot \bar{r}}, \quad \bar{B}_{i}=B_{0} \bar{u}_{\phi} e^{-i \bar{k} \cdot \bar{r}}
$$

For $\mathrm{H}$-receive and $\mathrm{V}$-receive modes we define $\Sigma(* H)=$ $-\bar{u}_{\theta} \cdot \bar{\Sigma}$ and $\Sigma(* V)=\bar{u}_{\phi} \cdot \bar{\Sigma}$ respectively. In [10] we used the tangent plane approximation for the surface fields in (77) to get the following expression:

$$
\begin{aligned}
\Sigma(p o l)= & \frac{i k^{2}}{k_{z}}\left[2 R(p o l)-\sin 2 \theta R_{1}(p o l)\right] C\left(2 \bar{k}_{H}, 2 k_{z}\right) \\
& -4 \Theta(p o l) k^{2} \sin ^{2} \theta R(p o l)^{2} D\left(2 \bar{k}_{H}\right)
\end{aligned}
$$

where pol $=H H$ or $V V, \Theta(H H)=-1, \Theta(V V)=+1$, $R(H H)=R^{T E}, R(V V)=R^{T M}, R_{1}(H H)=R_{1}^{T E}$, $R_{1}(V V)=R_{1}^{T M}$ and

$$
\begin{gathered}
C\left(\bar{k}_{H}, k_{z}\right)=\int d^{2} \bar{x} e^{-i \bar{k}_{H} \cdot \bar{r}-i k_{z} \eta} \\
D\left(\bar{k}_{H}\right)=\int d^{2} \bar{x} e^{-i \bar{k}_{H} \cdot \bar{r}} \eta(\bar{x}) .
\end{gathered}
$$

To calculate the curvature corrected root RCS we must use (48) to supply corrections to the tangent plane expressions and then insert the resulting quantities into (77). Note however that equation (48) represents a constraint between the values of the electric and magnetic fields on the surface. It does not tell us what values of the electric and magnetic fields should be inserted and precisely how the curvature correction should be applied. Here we make the assumption that the magnetic field is given by the tangent plane approximation and that the electric field is obtained through (48). This is consistent with the views expressed by Mitzner on this matter [11].

\section{B. Corrections to the root RCS}

As discussed above, in the following analysis we assume that $\bar{n} \wedge \bar{B}_{1}$ is given by the reflection-coefficient formula (79) and $\bar{n} \wedge \bar{E}_{1}$ is given by the sum of two terms, the first of which is given by the reflection-coefficient model (78) and the second of which is a curvature correction of the form:

$$
\frac{1}{c}\left[\bar{n} \wedge \bar{E}_{1}\right]^{C}=\frac{1}{i k_{1} N^{2}} \overline{\bar{R}} \cdot \bar{n} \wedge\left(\bar{n} \wedge \bar{B}_{1}\right)
$$

Using equation (79) one can show to lowest order in $\bar{\nabla} \eta$

$$
\begin{gathered}
\bar{n} \wedge\left(\bar{n} \wedge \bar{B}_{1}\right) \sim\left[1-R^{T E}\right]\left(\bar{e}_{z} \cdot \bar{B}_{i}\right) \cot \theta \bar{e}_{\alpha} \\
-c^{-1}\left[1-R^{T M}\right]\left(\bar{e}_{z} \cdot \bar{E}_{i}\right) \csc \theta \bar{e}_{\beta} .
\end{gathered}
$$


Let us now consider H-transmit where $\bar{e}_{z} \cdot \bar{E}_{i}=0$ and $\bar{e}_{z} \cdot \bar{B}_{i}=$ $-B_{0} \sin \theta e^{-i \bar{k} \cdot \bar{r}}$. Then using expression (72) for the $R$-matrix, we get the following corrections to the electric surface field through (85)

$$
\begin{gathered}
\frac{1}{c}\left[\bar{n} \wedge \bar{E}_{1}\right]^{C} \sim \frac{i B_{0}}{2 k N^{2}}\left[1-R^{T E}\right] \cos \theta e^{-i \bar{k} \cdot \bar{r}} \times \\
{\left[\left(\eta_{y y}-\eta_{x x}\right) \bar{e}_{x}-\eta_{x y} \bar{e}_{y}\right] .}
\end{gathered}
$$

The curvature correction to $\Sigma(* H)$ is given by

$$
\Sigma^{C}(* H)=\frac{i k}{c B_{0}} \int d S e^{-i \bar{k} \cdot \bar{r}}\left[\bar{u}_{\theta}-e^{2 i k_{z} \eta} R^{T E} \hat{\bar{u}}_{\theta}\right] \cdot\left[\bar{n} \wedge \bar{E}^{C} .\right.
$$

Therefore, in the LSV approximation, the curvature correction to the root RCS for $\mathrm{HH}$ is:

$$
\begin{aligned}
\Sigma^{C}(H H)= & -\frac{1}{2 N^{2}}\left[1-R^{T E}\right] \cos ^{2} \theta\left\{G\left(2 \bar{k}_{H}, 2 k_{z}\right)\right. \\
& \left.-4 k^{2} R^{T E} \sin ^{2} \theta D\left(2 \bar{k}_{H}\right)\right\}
\end{aligned}
$$

where

$$
G\left(\bar{k}_{H}, k_{z}\right)=\int d^{2} \bar{x} e^{-i \bar{k}_{H} \cdot \bar{r}-i k_{z} \eta}\left[\eta_{y y}-\eta_{x x}\right]
$$

and integration by parts has been used to give

$$
\int d^{2} \bar{x} e^{-2 i \bar{k}_{H} \cdot \bar{r}}\left[\eta_{y y}-\eta_{x x}\right]=4 k^{2} \sin ^{2} \theta D\left(2 \bar{k}_{H}\right) .
$$

A similar argument leads to the curvature correction for the VV root RCS:

$$
\begin{gathered}
\Sigma^{C}(V V)=\frac{1}{2 N^{2}}\left[1-R^{T M}\right]\left\{G\left(2 \bar{k}_{H}, 2 k_{z}\right)\right. \\
\left.-4 k^{2} R^{T M} \sin ^{2} \theta D\left(2 \bar{k}_{H}\right)\right\} .
\end{gathered}
$$

\section{SPM limit}

In this sub-section we look at the consequences of including the first curvature corrections in the root RCS to the agreement of our half-space model with the small perturbation method (SPM). In [10] we obtained the following expansions for the tangent plane approximation:

$$
\begin{aligned}
& \Sigma(H H) \sim-4 k^{2}\left(1-\sin ^{2} \theta\right)\left[1-2 \cos \theta N^{-1}\right. \\
& \left.\quad+2\left(\cos ^{2} \theta-\sin ^{2} \theta\right) N^{-2}\right] D\left(2 \bar{k}_{H}\right)+O\left(N^{-3}\right) \\
& \Sigma(V V) \sim-4 k^{2}\left[\left(1+\sin ^{2} \theta\right)\left(1-2 \sec \theta N^{-1}\right)\right. \\
& \left.+2 \sec ^{2} \theta\left(1+2 \sin ^{2} \theta\right) N^{-2}\right] D\left(2 \bar{k}_{H}\right)+O\left(N^{-3}\right)
\end{aligned}
$$

which agreed with Valenzuela's perturbation results only to $O\left(N^{-1}\right)$. In the SPM limit, we have:

$$
G\left(2 \bar{k}_{H}, 2 k_{z}\right) \rightarrow 4 k^{2} \sin ^{2} \theta D\left(2 \bar{k}_{H}\right)
$$

so that using $R^{T E}, R^{T M}=-1+O\left(N^{-1}\right)$ means that the curvature corrections given by (89) and (92) expanded to order $N^{-2}$ are

$$
\begin{aligned}
& \Sigma^{C}(H H) \sim-8 k^{2} N^{-2} \sin ^{2} \theta\left(1-\sin ^{2} \theta\right) D\left(2 \bar{k}_{H}\right) \\
& \Sigma^{C}(V V) \sim 8 k^{2} N^{-2} \sec ^{2} \theta \cos ^{2} \theta \sin ^{2} \theta D\left(2 \bar{k}_{H}\right) .
\end{aligned}
$$

These are precisely the corrections necessary to improve the agreement of the half-space model with SPM to $O\left(N^{-2}\right)$. Thus we have the following result. If the dielectric halfspace Green's function is used together with the raw tangent plane approximation, expressed by a reflection coefficient model, we obtain a model of the root RCS that agrees with SPM to $O\left(N^{0}\right)$ and $O\left(N^{-1}\right)$ when the surface perturbations become small. If we incorporate curvature corrections into the boundary conditions, in their simplest possible form (first order Rytov model), then the agreement is improved to include terms $O\left(N^{-2}\right)$.

\section{CONCLUSIONS}

We have shown how to derive high-order IBCs using a general vector basis adapted to the surface of a high-contrast dielectric. This removes the need to construct principal curvature axes or an orthogonal curvilinear coordinate system. As an example, we have derived the form of the IBC for a surface characterized by a Monge patch. This has been applied to the problem of scattering from a deformed plane using a dielectric Green's function, and we have shown that the use of a curvature-corrected IBC improves the agreement between dielectric half-space theory and SPM. We believe the high-order IBC in the form we have given can be used in a significantly larger category of scattering geometries than has hitherto been practical.

\section{ACKNOWLEDGMENTS}

Oxford System Solutions (now Cymath Limited) carried out this work under contract to the UK Defence Evaluation Research Agency. Thanks are due to A. Jolly, T. LamontSmith, B. C. Barber, R. J. A. Tough and K. D. Ward for helpful discussions, and to our anonymous referees for several helpful comments.

\section{REFERENCES}

[1] J. A. Ogilvy, Theory Of Wave Scattering From Random Rough Surfaces, Adam Hilger, Bristol, Philadelphia and New York, 1991.

[2] D. Holliday, G. St-Cyr and N. E. Woods, "A radar ocean imaging model for small to moderate incidence angles", Int. J. Remote Sensing, vol. 7, no. 12 , pp. 1809-1834, 1986

[3] G. Pelosi and P. Ya. Ufimtsev, "The Impedance Boundary Condition," IEEE Antennas and Propagation Magazine, vol. 38, no. 1, pp. 31-35, Feb. 1996.

[4] A.N. Shchukin, Propagation of Radio Waves, Publishing House Svyazizdat, Moscow, 1940.

[5] M. A. Leontovich, Investigations on Radiowave Propagation, Part II, Printing House of the Academy of Sciences, Moscow, 1948.

[6] S. M. Rytov, "Calcul du skin-effet par la méthode des perturbations," J. Phys. USSR, vol. 2, p. 233, 1940.

[7] J. L. Volakis and H. H. Syed, "Application of higher order boundary conditions to scattering by multilayer coated cylinders," J. Electromagn. Waves Appli., vol. 4, no. 12, pp. 1157-1180, 1990.

[8] T. B. A. Senior, "Approximate boundary conditions for homogeneous dielectric bodies," J. Electromagn. Waves Appli., vol. 9, no. 10, pp. 1227 1239, 1995.

[9] T. B. A. Senior and J. L. Volakis, Approximate Boundary Conditions in Electromagnetics, IEE Electromagnetic Waves Series 41, London: IEE Press, 1995.

[10] W. T. Shaw and A. J. Dougan, "Green's Function Refinement as an Approach to Radar Backscatter: General Theory and Applications to LGA Scattering from the Ocean," Trans. Antennas Propogat., vol. 46, no. 1, pp. 57-66, Jan. 1998 
[11] K. M. Mitzner, "An Integral Equation Approach to Scattering From a Body of Finite Conductivity," Radio Science, vol. 2, no. 12, pp. 14591470, Dec. 1967.

[12] M. Artola and M. Cessenat, "Diffraction d'une onde électromagnétique par un obstacle borné à permittivité et perméabilité élevées," C. R. Acad. Sci. Paris t. 314, Série I, pp. 349-354, Feb. 1992.

[13] C. L. Holloway and E. F. Kuester, "Impedance-Type Boundary Conditions for a Periodic Interface Between a Dielectric and a Highly Conducting Medium”, Trans. Antennas Propogat., vol. 48, no. 10, pp. 1660-1672, Oct. 2000.

[14] H. D. Ngo and C. L. Rino, "Application of beam simulation to scattering at low grazing angles 1. Methodology and Validation," Radio Science, vol. 29, no. 6, pp. 1365-1379, Nov.-Dec. 1994.

[15] G. R. Valenzuela, "Depolarisation of EM waves by slightly rough surfaces," IEEE Trans. Antennas Propagat., vol. AP-15, no. 4, pp. 552557, Jul. 1967. 